\title{
The Historical Evolution Research of Information Interaction Design
}

\author{
Yangshuo Zheng \\ Academy of Art \& Design, Tsinghua University, Beijing, China \\ zhengyangshuo@163.com
}

\begin{abstract}
This paper focuses on three parts: first, based on interdisciplinary, summary and expand the information interaction design concept and connotation system, clearer information interaction design system and constitute essential characteristics; Second, original build the information interaction design system thinking model in four dimensions "environment -human --technology--objects", launched research on social environment context, psychological needs of information users, Information technology research, information interaction design products; Third, combined and summarized information interaction design thinking model in existence and characteristics way of various forms of society, from the perspective of society evolution form the essence analysis of information interaction design, explored the transformation process of correspondence between information interaction design and the development of society patterns.
\end{abstract}

Keywords: information Interaction Design, Historical evolution, Information society.

\section{Background}

With the continuous progress of science and technology, information and explosive expansion of knowledge has become the core of today's social life. In the mid-20th century, the outbreak of a third world scientific and technological revolution, that the information technology revolution. Its Essence and core is the development of the information science and technology, including information related to human understanding of information understanding, information creation and dissemination of information, covering a very wide range. IT revolution involving either from the scope and scale of scientific thought or innovation disciplines are much higher than the previous agricultural revolution and the industrial revolution. Can be easily found that, we are experiencing the information technology revolution in human history to bring the greatest period of change. This information technological revolution is not only to showcase the vision of a new world, and to construct a new scientific way of thinking, that is-information thinking.

With the number of worldwide microcomputer persistent rapid increase in the increasing popularity of the Internet and wireless communications to Information 
Technology, information technology revolution makes people's life has changed dramatically, and it brought to mankind is not only science great progress on the technical level, but also to enhance the whole level of information society, has been fully reflected in the mode of production, lifestyle, fashion and other sensible perspective. The rapid development of information technology has become a huge impetus to the economic development of the society, information technology revolution and human life has been closely together. Basis of the information technology revolution is the great development of computer and network technology, and huge information can now be digitalized. digitalized information promote the rapid development of the information technology revolution, and triggered a series of changes. Massachusetts Institute of Technology professor and director of the Media Lab, Negroponte was in his monograph " Being Digital, " which said that the development of information technology will change the way humans learn, work, entertainment, namely, information technology change the human way of life. "Digitized so that the information can instantly reach the other side of the world, so that people experience a sense of closeness to the world of space, so as to promote the progress of human civilization. " Negroponte in 1995 for the development of information science and technology have made academic thesis has become a reality today. Human learning, work, entertainment or even the whole way of life have undergone tremendous changes in the way we perceive the world also undergone tremendous changes, which almost are closely related to the information technology revolution.

Today, a wide range of information is not only ubiquitous in life, and has become an important part of the social operation. IT revolution has made the depth and breadth of information dissemination has been greatly improved, each user access to information and means of transmission of information has become increasingly rich, convenient and quick. Digitalized information allows us to use a simple binary code " 0 " and "1 " to achieve the sound, text, pictures, video and other types of data coding and decoding ; all kinds of information processing, storage and transmission are achieve standardization and high speed processing mode of the network, which greatly enhances the ability of human information processing and transmission of information. IT innovation has spawned countless new digital information products, the Internet has developed into a variety of applications that can accommodate advanced information platform. With the further development of the information technology revolution, the future of the lifestyle, information transmission mode, the user emotional demand will continue a series of new development and change.

\section{Significance: A Systematic Theoretical Research Thoughts}

Information interaction design is cross-disciplinary integration research direction of design disciplines and humanities, information engineering disciplines, and etc. Information interaction design based on In information science, organizational behavior, interdisciplinary research biomechanics, kinematics, physiology, automatic control theory makes the user experience as the core, with the digitization of 
information collection analysis and statistical techniques as a reference, finally to expand the experimental studies on the information product interacts with the environment, explore human-machine sensors, interactive, human-computer interaction and other information interactive mode works. The development of information interaction design has an important-looking and leading role for the survival and development of design discipline. With the continuous development, the design theoretical study also presents new features, it is no longer confined to the words of pure theory, but increasingly focuses on practical applications for design guidance and reflection. Information Interaction Design is one of the most typical representative, it embodies the direction of today's latest development and application of design.

This article will try to build the research framework for information interaction design, using the theory and methods of multidisciplinary scientific and comprehensive study is a great research potential and challenging. From the social history and cultural heritage point of view, reconstruct the cognitive knowledge of information interaction design, exploring various forms of information interaction design on specific applications through the whole development of society; construct "environment human - technology - objects "systems thinking, comprehensive and systematic analysis of the various constituent elements and parse" environment - human technology - objects "relationship between the various elements of the system, and ultimately restore the essence of design behavior:" Not only is a creative activity, but also for humanity creating a more rational, more healthy lifestyle production and to build a sustainable and harmonious society. "

\section{About Information Interaction Design}

\subsection{Basis of Information Interaction Design}

The establishment and development of information interaction design is based on the "information technology". UNESCO makes definition of Information Technology: " Used in information processing and processing of science, technology and engineering training methods and management skills; The above methods and techniques of application; Computer and its interaction with people, machine; Corresponding social, economic, culture and almost various kinds of things. "Researchers Don Schultz and Philip Kitchen define IT (information technology) as " can make human knowledge, data and experience on a global scale organizations or between individuals a simple, fast transfer of equipment, technology and performance integrated. It is a way to include a variety of new electronic data storage, transmission, distribution and analysis constituted, not only contains various forms of common tools, further including the most sophisticated forms of mathematical analysis and calculation methods." Although there is no very clear uniform definition of IT, but their essence is a way to extend and expand the means and methods of human information functions.IT revolution is rapidly changing information media and its environment, IT becomes technical support almost all media composition, technical standards and development orientation, become the most popular medium of information interaction on new meaning. 
On the perspective of design disciplines, in the computer, Internet and other information tools has been more deeply universal applied today, research width and depth of information interaction design research has been greatly expanded. Information interaction design primarily study the way of human society and the transmission of information and get responding information, ultimately to establish contacts with the outside world. Since it adequately reflects the tendency of service design for users, therefore, information interaction design should be part of an important branch of "Information Science and social studies" field.

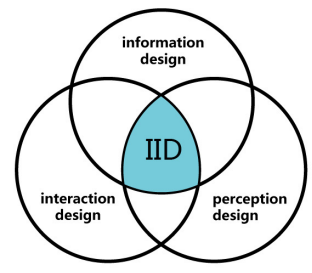

Fig. 1. Concept of information interaction design

\subsection{Concept of Information Interaction Design}

Research subject of this paper, "Information interaction design" is actually a systematic cognitive design fields, composed of the three design direction: information design, interaction design, perception design. As early as 1999, Shedroff published papers pointed out that should the information design and interaction design considerations together, treat it as a unified field of design theory. Seedorf argued that the information interaction design is integrated consisting of "information design", "interaction design" and "perception design", Shedroff call it "Information Interaction Design". Information Interaction Design should be designed to standardize and facilitate information-oriented mode of human interactions with the theoretical prototype, the focus should be to build more rational human information interaction and corresponding conduct under the information society background. As can be seen, Information Interaction Design reflects the design trends in information society with its own attributes in accurate way, which can be the most cutting-edge contemporary design direction is inevitable.

Information Interaction design is about the artifact objects research, the main contents include a new generation of natural harmony of man-machine dialogue mode, the language of communication design, product design principles, information services, software application mode, no interface design technology, HCI design Principles and etc. As a design discipline has a strong social application, Information Interaction design can real-time reflect the information technology development and application trends, thus facilitate communication between user and the outside world in an innovative way. Information interaction design itself is in a continuous integration and rapid development process in order to adapt the changing environment. Information interaction design emphasis on expanding and extending from the " non-material " perspective, through information communication, information 
interaction, information applications and information services. It should be emphasized that information interaction design is not a new design concept in an absolute sense, but as a integrated result of the development of history, advances in technology, human improvement of consciousness thinking.

In the "Information Interaction Design" concept system framework, "information design" is not a substitute for graphic design or visual communication design tool, but to provide a framework for interactive information, organization and forms the main concern of the data. " Interaction design " is a symbol of "creating and telling stories ", focusing on how to use reasonable skill and application, creating a pleasant user experience, which through across the field of art and information technology. "perception design " is a harbinger of future trends and directions of IT development. Perception design studies information possibilities, determine how can the information interaction and information application create reasonable way, as how deep and wide of the information dissemination. Therefore, "Information Interaction Design" have a systematic, research -based holistic basis: Information Design focuses on the level of information to convey, Interaction design focuses on interactive technology to achieve the design goal, perception design focuses on the user's emotional and demand levels ; these three directions composed a systematic theoretical design system.

\subsection{System Model of Information Interaction Design}

On the basis of the Information Interaction Design concept, I created a "environmenthuman - technology - objects" system model of Information Interaction design. "environment", "human" constitute the external environment of information interaction design activity; "technology", "objects" constitute the internal environment, including the development and application of information technology, the concept of settings and details of products. On the one hand you can understand the "environment", "human", to grasp the macro direction of information interaction design; On the other hand, through the organization and expression of the "technology", "objects", to grasp the micro realization of information interaction design approach. External and internal environment can be seen as a parallel relationship, integration and balance between them is the key of information interaction design.

In short, the existence and development of information interaction design, is the relationship between its environment, human, technology, and objects; either from the perspective of user-oriented design, but also from the information activity with its environmental point of view are true.

Relationship between information interaction design and environment, mainly refers to the relationship with the social environment. Phenomenological theory thought that the environment is not an abstract place, but by the specific things that the composition as a whole. Information interaction activities require different environments as a basis, in order to facilitate information interaction activities generate. We can say that environmental factors determine human behavior, determines the attributes of the information interaction design. Information interaction design activities and environmental linkages, provided its hold in the environment of the significance of a particular role. Through this research perspective, information 
interaction design activity embodies the value of user functionality in the social environment, which provide the information for the application, but also reflects the cultural values in the process of social development. In the system model, environmental products (dominant presence), user (intellectual existence) and culture (invisible presence) nicely connects together.

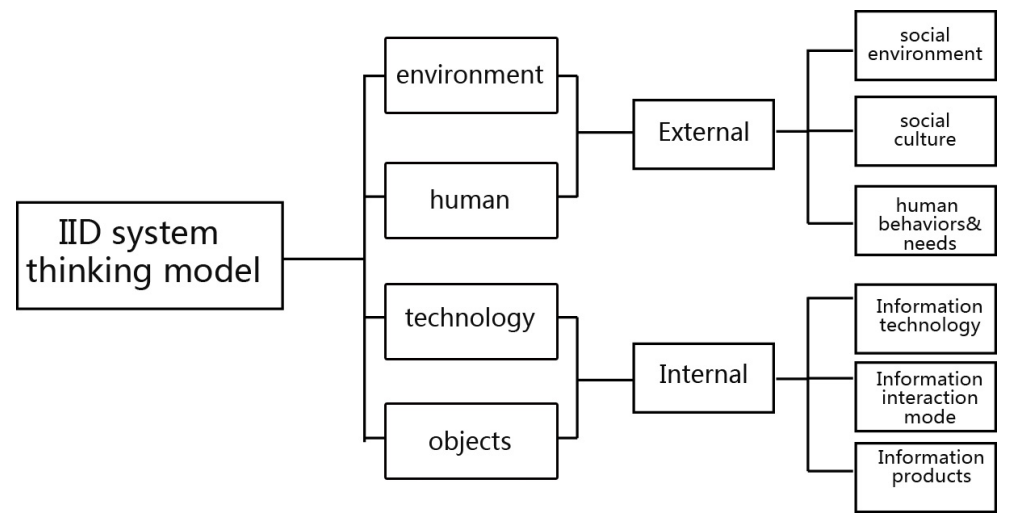

Fig. 2. IID design system thinking model

Relationship between Information interaction design and human, mainly refers to users of user-oriented design. In a sense, Information interaction design is based on users' creative process, and its purpose is to help users solve problems, improving the user experience, and then realize the target audience emotional resonance. From this perspective, this relationship includes applications of sensory experiences (how to use visual, auditory, tactile, olfactory, gustatory perception meet user needs), the user's mental model and user behavior. Information interaction design From the "Design for others" to "Collaborative others for Design" is the most unusual place with other design, to some extents, users can determine even the direction of design. future users will be involved in the design, become collaborators, eventually decision-makers. Therefore, from the study of "usability", "ease of user" extends to how to play to users' "initiative", "creative", will be key to the future development of Information interaction design.

Relationship between information interaction design and technology, mainly refers to the relationship between design activities and information technology. The traditional design perspective, often makes "technology" and "objects" as a whole, which leads to neglecting the real technology. IT does not seem only simple tools, information technology for the design often have a decisive significance and effect. Kevin Kelly even claimed that "technology is the seventh existence of life. Technology is an extension of life, rather than something separate and life beyond." In fact, every technology innovation will make technical elements improved, bring new opportunities and new changes and diversity final design mode.IT will be a key factor in promoting the progress of information interaction design, makes predicting the future development trend of information interaction design possible. 
Relationship between information interaction design and objects, mainly refers to the intrinsic relationship between the specific interaction information to convert the design process and results, and its essence is an internal agreement of contact. The development of information technology has given the diversity of forms of objects (material and non-material), but from the perspective of artificial fact is concerned, the matter is still mentioned belong to "artifact" category, which determines the type of objects. Information interaction design reflect features mostly achieved through the "objects" as the carrier. Thus, many of the traditional design for the "objects" rule, characteristics, cognitive attributes in the information interaction design still have in common.

In the previous design research study, most design emphasize form design while ignoring the inherent design factors associated even with the history, culture and other factors discussed in the background, showing the limitations and the lack of a more overall systematic theoretical research ideas. Therefore, from the perspective of the design system model presented in this paper to think about the information interaction design, will make a broader, more holistic, more accurately grasp the development context and the law of information interaction design activities, then carry out exploration and innovation of information interaction design in future.

\section{Historical Evolution Research of Information Interaction Design}

Human society is constantly moving forward, information interaction design also will continue to move forward. We can say that the development of human society to move forward with information interactive design is synchronized at every stage of historic evolution, social development are accompanied by great changes in information interactive design.

\subsection{Primitive and Agricultural Society}

Ever since mankind went out of the forest, tribal clan is relying gregarious subsistence hunting and gathering together, this is because in primitive society the harsh natural environment, human needs through collaborative social way to live together to overcome individual weaknesses. Dissemination of information and the development of human society is synchronized. We can say that gregarious subsistence model is the foundation of human information dissemination, burrowing cultural history is the earliest history information dissemination.

From a macro broad view of design history development, the human birth, the technology birth, and then culture birth, therefore it can be said that design birth. In Primitive society, human clearly understood that struggling with nature, the ability of human beings is very limited. In agricultural society, agricultural and animal husbandry means the relationship between humans and the natural world level had been leaped, on behalf of human life from relying on natural adaptation sources, change and transformation for the use of natural productivity, production level has been largely 
increased. The production relationship in agricultural society is a relatively expanded reproduction mode, the technological level compared to the later industrial society is still low, still reflects a strong natural features.

From the information interaction design perspective, in primitive society, orality to the original human society has far-reaching significance, it essentially is a major social activity of people, thus forming a culture of orality. Before oral communication, human only through body movements and simple facial expressions, vaguely describe their desired information expressed. Oral communication is the major source of social information interactively to improve the human understanding, and improve the ability to adapt and transform nature, to enhance the human emotion, memory and thinking level. In agricultural society, the invention of papermaking and printing made human extending the visual ability, and promoted the development of social productive forces, gradually formed a society of printing culture, represented the arrival of the substance for the information age. A number of people work specializes in the dissemination of information, knowledge, ideas and a wide range of information start quickly and spread widely in the crowd, a symbol of an epoch-making power of the human society development. Papermaking and printing enhances the understanding of the human experience and the ability to grasp the whole world. Although it is impossible to make information generated input and output in interaction synchronization peer, but has liberated the dissemination of information to the inherent limitations of space -based printing information interactively, it can save both time and information transmitted across space constraints, given the abundance of information may be copied.

In the period of primitive society and agricultural society, the development of information interaction mode from facing the information interactively gradually evolved into the form of written text-based separation behavior, the evolution of information interaction mode allows the sender and receiver of information is no longer necessary limitations at the same time and place to complete information interactions. Although the information interaction mode in primitive and agricultural society is relatively simple, and has a strong regional characteristics; but for the human spirit to enjoy the social life and cultural transmission, provides the necessary conditions for the material basis for the development of world science and culture has played a huge role. Following the text, the subsequent development of information interaction mode also continue the trend to extend the message sender and receiver separated and play spatial characteristics, in the existence of a more diversified approach.

\subsection{Industrial Society}

For society under the great influence of the industrial revolution, all kinds of people start quickly production of creation, production, human greatly improved understanding of the material world and transform ability. The industrialization of the social development has made remarkable achievement, makes the human can fully enjoy the energy resources and material resources, all kinds of machine effectively expanded the human body, promote the development of social productivity. And the great change of information technology in the industrial society has also promoted the information interaction mode forward. 
In industrial society, human interaction in the past information uncertainty, geographic limitations, low efficiency has been improved to a large extent, the value and importance of information dissemination have increasingly been revealed. Telephone, telegraph, radio and television, etc. These great invention are enough to load the history of human civilization, finally shorten the distance between people, speed up the society develop pace, improving the people's information communication efficiency. Telephone represents the ability to extend the range of human hearing, radio and television represents the human auditory and visual ability diversify; variety of new information technologies continue to invent and popularize of carrier applications, interactive way to help the development of information interaction mode. Electronic equipment as an information interactive medium has become the mainstream of society, which has a very distinct and accurate, efficient features, information interactive activity has basically no longer limited by traditional factors of time, space and so on. Quality and efficiency of information dissemination during the industrial society has made great progress in the emotional level and technical level have a great impact on information users, and its breadth and depth compared to agricultural society has been a huge improvement.

Overall, the information interaction mode in industrial society, although expression in the form of information and user information dissemination process perceived need at the technical level has been greatly improved, but for the individual in terms of information interactive, comprehensive grasp of the overall demand for information has not been fully met. For the system concept of information interaction design, in industrial society period, the completion of information design were ahead of interaction design and perception of design. unidirectional dissemination of information were still the vast majority, the process of information dissemination in time and space were spatially separated, there has primary and secondary in the order, and existed a certain degree of time cost. Mainly in the lack of feedback and loop of information dissemination, ignored the psychological complexity of information user, inadequate grasp the relationship between the whole and the constituent elements of information dissemination.

\subsection{Information Society}

Information society is the social forms of rapid development of information technology and widely used as main indicator. In information society, information have been rapidly accumulating, transmitting, storaging, the value of information has been fully played, creating wealth for human society and to some extent, improve the people's living environment. Information Society represents a new transformation of human civilization.

Information society means new information economy generated, based on a universal application of computer and Internet, reducing the time and space for the entire production process society. Information interactive content of the information society through the spread of bits in the form of raw data, eventually revert to the form of information via communication networks; bits can be produced anywhere and at any time. Compared to traditional interaction mode like newspapers, radio, television and 
other, Internet highlights the more rapid, convenient and efficient features and is suitable for two-way and multi-directional information interaction. Information Society integrates of a variety of information interaction forms, eventually gave rise to pleasant user experience. "We media" as the representative of the new information interaction product, brings personalized information and high-speed information transmission speed and extremely timely, bring people high-quality information services and information experience.

Information technology have driven the cost of production of information products decreased, product replacement cycles are getting shorter. Since the development of information technology for internal information -driven, relevance of information interactive products will be the main concern of information interaction design goal. In essence, the biggest change from industrial society to information society is reflected in the economic structure of society, from production economy to service economy, which human is the most important. Firstly, the features of user needs began quietly changing, design in the pursuit of product features, while increasing emphasis on diverse forms of cultural diversity. Secondly, the design objects began to shift to information services, information technology is the changing basis; For more information on the service requirements of personalized attention can customize content and interactive user experience, a new design and new content service system may arise. Furthermore, the design content is designed from a single or a single series to a system design. in information society, the content has been designed from the object itself, turning on the functional design objects, people, society, culture various elements of the system the overall relationship between system design.

In summary, information society create a new context environment for information interaction design, both the authenticity of the existence of the real world, and the virtuality of the online world. Information interaction design is essentially an act of creation, through induction and integration of the relevant elements of whole system, guide the user to build more innovative reasonable information interaction activity.

Table 1. Comparison of IID between different societies

\begin{tabular}{|l|l|l|l|}
\hline & $\begin{array}{l}\text { Primitive and } \\
\text { Agricultural society }\end{array}$ & Industrial society & Information society \\
\hline Core elements & Natural objects and \\
\hline $\begin{array}{l}\text { Technological } \\
\text { revolution }\end{array}$ & Agricultural revolution & Industrial revolution & $\begin{array}{l}\text { Information } \\
\text { knowledge } \\
\text { revolution }\end{array}$ \\
\hline $\begin{array}{l}\text { Symbolic } \\
\text { Inventions }\end{array}$ & Bronzes and iron & steam engine & $\begin{array}{l}\text { Computer } \\
\text { Internet }\end{array}$ \\
\hline $\begin{array}{l}\text { Economy } \\
\text { Features }\end{array}$ & $\begin{array}{l}\text { Acquisition of natural } \\
\text { resources }\end{array}$ & $\begin{array}{l}\text { Production of material } \\
\text { resources }\end{array}$ & $\begin{array}{l}\text { Information services } \\
\text { based on knowledge }\end{array}$ \\
\hline culture & Regional & Globalization & Diversity \\
\hline $\begin{array}{l}\text { Human needs } \\
\text { Survival needs }\end{array}$ & $\begin{array}{l}\text { Possession of material } \\
\text { requirements }\end{array}$ & $\begin{array}{l}\text { Spiritual } \\
\text { needs }\end{array}$ \\
\hline $\begin{array}{l}\text { Information } \\
\text { Characteristics }\end{array}$ & Closed & One orientation and open & Interaction and open \\
\hline
\end{tabular}




\subsection{Evolution of Information Interaction Design}

In any form of society, there exists communication needs for people anywhere, which determines the general society have the necessity of information interaction activities. Evolution of information interaction design is a process of evolution, the development speed and completeness of information dissemination in a social environment, in a sense marks a level of civilized society.

In primitive and agriculture society, since the text had appeared, in the true sense of mankind from the beginning of information dissemination, papermaking and printing makes the emergence of information communication began to popularity. Limited to the historical period, information dissemination only had slow speed, the content is also relatively simple, can only be passed with relatively simple text and symbol message information.

In industrial society, dissemination mode of information had turning to mechanization and electronic communication type, information contents can be audio, video, images and other forms of integrated information. The invention of telegraph, telephone, radio, television means that the speed of information dissemination is close to the speed of light, which greatly accelerated the pace of development of human society. Information can be spread short distances, spread further expand the scope to further, eventually improved information transmission efficiency.

In information society, The information interaction mode are very diverse, both through traditional mass media ( such as face to face, phone calls, letters ) to expand information interaction activities, but also relies on new information technologies ( such as Email, Facebook, instant messaging, Internet phone ) Internet applications. The two sides continue to exchange information dissemination and reception of information through internet-based information media and supplement the information at any time and modify each other's feedback. Compared to the traditional way of information interaction, Internet-based information interactively contain a certain time cost, compared to face impression formed more slowly, but eventually can achieved with the same exchange effect like face to face. The existence of the Internet as information interaction activities, create an unprecedented space, network anonymity, motivate people new information applications, either from the expansion of the quantity or quality of people 's information interactions. From whatever perspective depth, breadth and interactive view of the extent, the information society are far better than in the past any form of society.

\section{Conclusion}

With the evolution of society history, information interaction design showed more penetration and influence on the development of society. Information interaction design not only meet the needs and desires of human life, but also increasingly coordinate with the objective nature and the human instinct, continuously enhance the quantity and quality of information interaction activities. Computers and Internet as a symbolic product of today's information technology, goes beyond the mere meaning of information tools; with its help, the relationship between humans and information 
society becomes more closely as human contact and social media. Compared with traditional designs, information interaction design can be seen as the extension of traditional design, but the essence is still the high level human thinking of how to achieve a more rational way of human living.

Maximum theoretical contribution of this paper is that from the perspective of the social development, did a number of in-depth research of information interaction design, combines and summarizes the evolution essence of information interaction design, linked it with the modern concept and traditional scientific knowledge system, clearly presented the development of information interaction design in theoretical way. This article highlighted that information interaction design not only exists in information society, but in the era of the information society becomes more clear to the past.

In short, the research of information interaction design and its "environment -human - - technology- -objects" design system thinking model, not only a macro thinking based on historical forms of social development characteristics, but also a design theory research innovation of information interaction design. By combing and summarizing the development of information interaction design evolution, the paper also shows the future direction of information interaction design development, information interaction design will become the most representative design branch of future development. I wish this study will bring more enlightenment for contemporary design research and the development of social sciences.

\section{References}

1. Liu, G.: Design Methodology, vol. 3. Higher Education Press, Beijing (2011)

2. LU Xiaohua:core of the media operations, http: //news.xinhuanet.com/newmedia/2005-01/07/content_ $2427865 . \mathrm{htm}$

3. Shedroff, N.: Information Interaction Design. In: Jacobsen, R. (ed.) A Unified Field Theory of Design in Information Design, pp. 267-292. The MIT Press, Massachusetts (1999)

4. Zhang, L.: Semiotic approach to product design, vol. 126. China Building Industry Press, Beijing (2011)

5. Kelly, K.: What technology wants. Preamble. CITIC Publishing House, Beijing (2011) 